\title{
Genome editing in wheat: exploration of new challenges for crop improvement
}

\author{
Kingsly J.N.B. \\ Department of Agriculture, School of Agriculture and Biosciences, Karunya Institute of Technology and \\ Sciences, Coimbatore, India \\ email: johnkingslyjk@gmail.com
}

Genome editing is one of the most efficient technique for wheat improvement. Wheat is considered as the most quotidian food for the mankind and there has been an urgent need to increase the yield of wheat in the next few years to sustain the human growing population. In the past few years, the wheat research has emphasized the fact that existing technology lacks to explain about the genetic transformation in wheat due to lack of knowledge of the genetic determinants underlying the traits that are responsible for its crop yield and quality. Recent breakthroughs in the wheat genome sequencing has provided a quantum leap in the wheat crop improvement. In this review, we outline the current progress in the genome editing for wheat crop improvement, genetic transformations, modification of the gene structures of the wheat and involvement of the expression of the site-specific nucleases in wheat for genome editing. We also explain about the new challenges that are underlying for the wheat crop improvement in exploiting the CRISPR/Cas9 complex. This review further abridges the fundamental gap enhancing the wheat yield, quality, nutritional value and resistances to various stress agents. 